\title{
GPPS-BJ-2019-0121
}

\section{IMMERSED BOUNDARY METHOD WITH MOVING HALF-DOMAIN BODY-FITTED MESHES FOR 3-D COMPRESSIBLE VISCOUS FLOW}

\author{
Jingyuan Wang \\ School of Jet Propulsion, \\ Beijing University of \\ Aeronautics and \\ Astronautics \\ wangjingyuan@buaa.edu.cn \\ Beijing, China
}

Peng Shan

\author{
School of Jet Propulsion, \\ Beijing University of \\ Aeronautics and \\ Astronautics \\ PShan@buaa.edu.cn \\ Beijing, China
}

\author{
Haitao Zhu \\ School of Jet Propulsion, \\ Beijing University of \\ Aeronautics and \\ Astronautics \\ zht83@126.com \\ Beijing, China
}

\section{ABSTRACT}

An Immersed Boundary Method combined with HalfDomain Body-Fitted Meshes has been proposed, in order to simulate three-dimensional viscous compressible flow through complex geometry objects such as propeller and propfan. Orthogonal Cartesian grids are adopted to simulate the mainstream, and the "body-fitted equidistant surface grids" immersed in the Cartesian grids are adopted to simulate the viscous flow near the surface. So that the mainstream could feel the effects of surface geometry exactly.

The equidistant surface grids generated by copying the object surface along the outer normal direction, are half-space constrained. It's easy and fast to automatically generate HalfDomain Body-Fitted grids, which have excellent orthogonality, and very suitable for viscous compressible unsteady flow simulation with turbulence model.

Strategies for finding contribution units and interpolating flow information have been introduced in detail. Flux Vector Splitting method and WENO-Z scheme were used for spatial discretization, while Runge-Kutta method for temporal discretization. S-A model was adopted for turbulence simulation. Numerical verification examples of both steady and unsteady flow are presented.

\section{KEY WORDS}

RANS Immersed Boundary Method, Overset meshes, Propfan/Propeller, WENO Scheme

\section{NOMENCLATURE}

$\begin{aligned} \alpha & =\text { angle of attack } \\ C_{P} & =\text { pressure coefficient } \\ C I & =\text { lift coefficient } \\ e_{t} & =\text { total internal energy } \\ F^{ \pm} & =\text {flux-split numerical convective fluxes }\end{aligned}$

$\begin{array}{cll}M a & = & \text { Mach number } \\ n & = & \text { rotate speed } \\ v & = & \text { kinematic viscosity } \\ p & = & \text { pressure } \\ R e & = & \text { Reynolds number } \\ \rho & = & \text { density } \\ T & = & \text { temperature } \\ \tilde{v} & = & \text { turbulence kinematic viscosity } \\ u, v, w & = & \text { velocity components } \\ (x, y, z, t) & =\text { Cartesian coordinate system } \\ (\xi, \eta, \zeta, \tau) & =\text { Orthogonal coordinate system }\end{array}$

\section{INTRODUCTION}

The generation of high-quality computational grids still takes up the main workload of the numerical simulation process. Although there are a lot of highly automated grid generation software available, it is still difficult to avoid tedious and boring grid generation, during design process of turbomachinery. Cartesian mesh method, which directly uses cubic meshes, is a very practical method for reducing the workload of fluid mechanical engineers. Cartesian Grid generation is fast, simple and highly automated. Immersed boundary method (IBM) is a very popular Cartesian grid computing method at present. Its core idea is to use two sets of grids, "Eulerian" and "Lagrangian", to respectively represent the fluid region and solid boundary, as shown in figure 1.

There are two types of common IBM. The explicit force source method, proposed by Peskin, extrapolates the Lagrangian force source term to a group of Eulerian fluid grids nearby, through a spatial discrete function. Then the force source term is directly substituted into the solving equations. Its advantage is convenient for application of force source, However, the accuracy is usually only first order at the boundary of surface. The implicit force source method, 
proposed by Tseng and Ferziger based on "Ghost-Cell", directly sets the velocity and pressure boundary conditions on the Ghost-Cell points inside the object surface (as shown in figure 1), thus avoiding the solution of the force source and effectively improving the resolution of the object surface boundary.

However, the shortcomings of ghost-cell method are very prominent. When dealing with 3-D complex shape surface, the algorithm for determining the location of the ghost cells is relatively complex and requires a large amount of calculation. The ghost-cell in the surface with sharp angle shape may correspond to multiple Eulerian flow field points, resulting in the decrease of accuracy. When applied to long and narrow shaped objects such as wings, there may be too few interior points and not enough ghost points to set boundary conditions. Limited by the minimum grid size, neither of two immersed boundary methods described above can accurately calculate the viscous forces at the boundary of the surface.

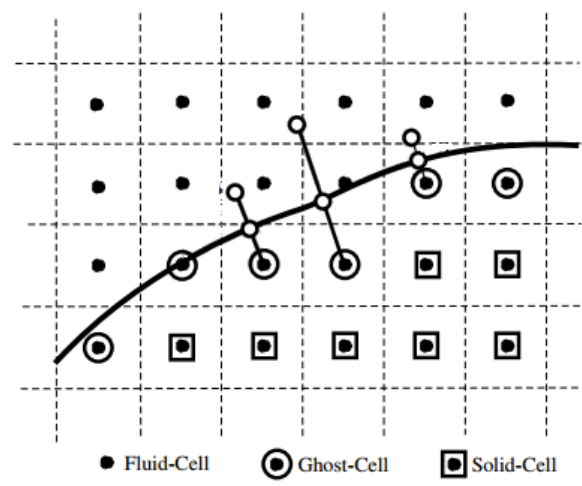

Figure 1 Ghost-Cell of Immersed Boundary Method

By analysing the advantages and disadvantages of the above two types of immersed boundary method, and combining contribution unit searching and interpolation technology in the overset grid method, this paper proposes a new immersed boundary method, Half-Domain Body-Fitted Mesh Immersed Boundary Method (HBM-IBM).

The strategy of "Half-Domain Body-Fitted Mesh Immersed Boundary Method" is similar to the "Ghost-Cell" method with implicit force source proposed by Tseng and Ferziger. The equidistant surface grids generated by copying the object surface along the outer normal direction, are halfspace constrained (as shown in Figure 4). The viscous flow sharply changed near the surface can be solved through the equidistant surface body meshes. Then the normal and tangential body force information will be interpolated into the orthogonal Cartesian meshes.

Due to the high shape resolution of the object surface, this method could achieve the same computational accuracy as the classical body-fitted grid method, and at the same time avoid the time-consuming and laborious generation of complex grids, highlighting the advantages of Cartesian mesh method.

When a body-fitted mesh moves, the former internal mesh point becomes the new Euler flow field point, and the accurate and correct flow solution will be obtained by interpolation from the body-fitted grids surrounding it, thus avoiding the defects in Ghost-Cell Method. The principle of this method is equivalent to the classical Ghost-Cell Method when the layer number of the half-domain body-fitted mesh is only one.

Due to the limited length, this paper summarized the implementation strategies of the HBM-IBM method, including the searching method for contribution units and the interpolation formula of conserved flux. The "governing equations", "spatial and temporal discretization schemes", "boundary conditions" and "turbulence model" used in the numerical simulation process were briefly introduced.

\section{HALF-DOMAIN BODY-FITTED MESH METHOD}

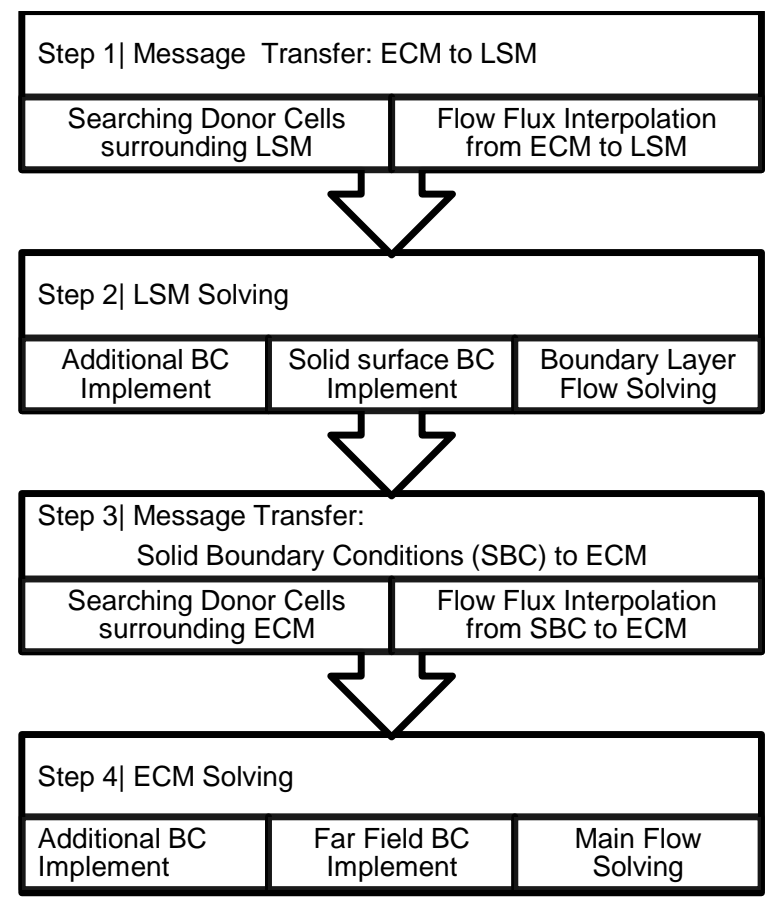

Figure 2 Main Steps of HBM-IBM

The main steps of HBM-IBM are shown in figure 2:

1) On one time-step, a suitable "contribution unit of Eulerian Cartesian Mesh (ECM)" surrounding Lagrangian Surface Mesh (LSM) is found, and the flow field information of ECM in the previous time step is interpolated into the LSM grids as the "additional boundary condition" of LSM;

2) Solving the boundary layer flow field near the object surface on the LSM, and then the information of the force on the object surface can be obtained;

3) Finding the "LSM contribution unit" surrounding the ECM, so as to apply the solid surface boundary condition and boundary layer viscous flow field information solved in the previous step, to the ECM through the spatial interpolation of flow flux;

4) Finally, the updated flow field information on ECM is used as the "additional boundary condition" of ECM to solve the flow field on the whole ECM grids, and the solution of this time-step is completed.

Hole-map method proposed by Chiu is used for searching contribution units. The adopted physical conservative flux interpolation method is the spatial second-order precision " 
Lagrange polynomial interpolation formula" proposed by Mittal, which is as follows:

$$
\begin{gathered}
\phi_{P}=f_{\phi}\left(\phi_{(i, j, k)}, \xi_{P}, \eta_{P}, \zeta_{P}\right) \\
f_{\phi}\left(\phi_{(i, j, k)}, \xi, \eta, \zeta\right)=\sum_{i=0}^{n} \sum_{j=0}^{n} \sum_{\substack{k=0 \\
C_{m}}} \phi_{(i, j, k)} C_{i}(\xi) C_{j}(\eta) C_{k}(\zeta) \\
m \in(i, j, k), \quad \prod_{\substack{l=0 \\
l \neq m}}^{n \in(\xi, \eta, \zeta), \quad \mathrm{\beta}-\beta_{l}} \frac{\beta}{\beta_{m}-\beta_{l}},
\end{gathered}
$$

Where, $\phi_{P}$ is the flux value at an interpolation point $\mathrm{P}$. $\left(\xi_{P}, \eta_{P}, \zeta_{P}\right)$ are the spatial interpolation coefficients at the point P. $\phi_{(i, j, k)}$ are the flux values at 8 vertices of contribution unit (shown in figure 3 ), and $C_{i}(\xi), C_{j}(\eta), C_{k}(\zeta)$ are the corresponding polynomial coefficients.

In equations 1 to 3 , the spatial interpolation is of order $(\mathrm{n}+1)$ precision. In order to reduce computational consumption, $\mathrm{n}$ is set as 1 in this paper. If necessary, a higher order Lagrange polynomial interpolation formula $(\mathrm{n}>1)$, can be used for higher precision requirements.

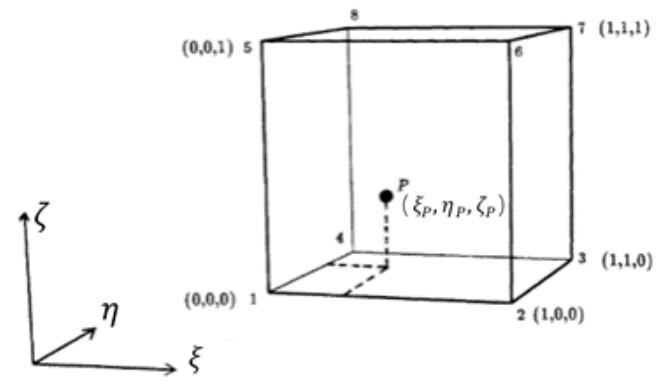

\section{Figure 3 Euler Contribution Unit Box Surrounding Surface Mesh Point $\mathbf{P}$}

As shown in figure 4, the principle of "HBM-IBM" method is introduced by taking "spatial position relation judgment" and "numerical flux interpolation" of ECM grid point Q, surrounded by LSM grids (L_BOX) as examples. Unlike the classical overset grid approach, in this method, all LSM grids only communicate with the ECM grids. The search of the contribution units and the interpolation of the flux are completed at the same step.

The above steps can be achieved through internal and external circulation:

In the outer loop, the minimum scope index of the E_BOX,$\quad\left(i_{\min }, j_{\min }, k_{\min }\right)_{\text {Ebox }} \sim\left(i_{\max }, j_{\max }, k_{\max }\right)_{\text {Ebox }}$ surrounding each LSM grid unit, was determined by traversal of all LSM grid points, and the "hole-map" method proposed by Chiu was adopted.

In the inner loop, the mesh points on the minimum index range E_BOX are traversed. Taking point $\mathrm{Q}$ in figure 4 as an example, the three-dimensional spatial coordinates of both point Q and 8 vertices of the contribution unit L_BOX, are substituted into equation (1) to obtain the nonlinear equations:

$$
\left\{\begin{array}{l}
x_{Q}=f_{x}\left(x_{(i, j, k)}, \xi_{Q}, \eta_{Q}, \zeta_{Q}\right) \\
y_{Q}=f_{\mathrm{y}}\left(y_{(i, j, k)}, \xi_{Q}, \eta_{Q}, \zeta_{Q}\right) \\
z_{Q}=f_{z}\left(z_{(i, j, k)}, \xi_{Q}, \eta_{Q}, \zeta_{Q}\right)
\end{array}\right.
$$

Newton - Raphson iteration method is adopted to solve nonlinear equations (4), and the spatial interpolation coefficient $\left.\left(\xi_{Q}, \eta_{Q}, \zeta_{Q}\right)\right|_{\text {Ebox }}$ is obtained. The value of this spatial interpolation coefficient can be used to judge the spatial position of point $\mathrm{Q}$. When condition:

$$
0 \leq\left(\xi_{Q}, \eta_{Q}, \zeta_{Q}\right) \leq 1
$$

is satisfied, the point $\mathrm{Q}$ on E_BOX must be inside the contribution unit L_BOX.

The flux on the contribution unit L_BOX is interpolated to the Euler mesh point Q located within it. By substituting the spatial interpolation coefficient of point $\mathrm{Q},\left.\left(\xi_{Q}, \eta_{Q}, \zeta_{Q}\right)\right|_{\text {Ebox }}$, into equation (1), the flux $\varphi_{Q}$ at point $\mathrm{Q}$ can be obtained as below:

$$
\varphi_{Q}=f_{\varphi}\left(\varphi_{(i, j, k)}, \xi_{Q}, \eta_{Q}, \zeta_{Q}\right)
$$

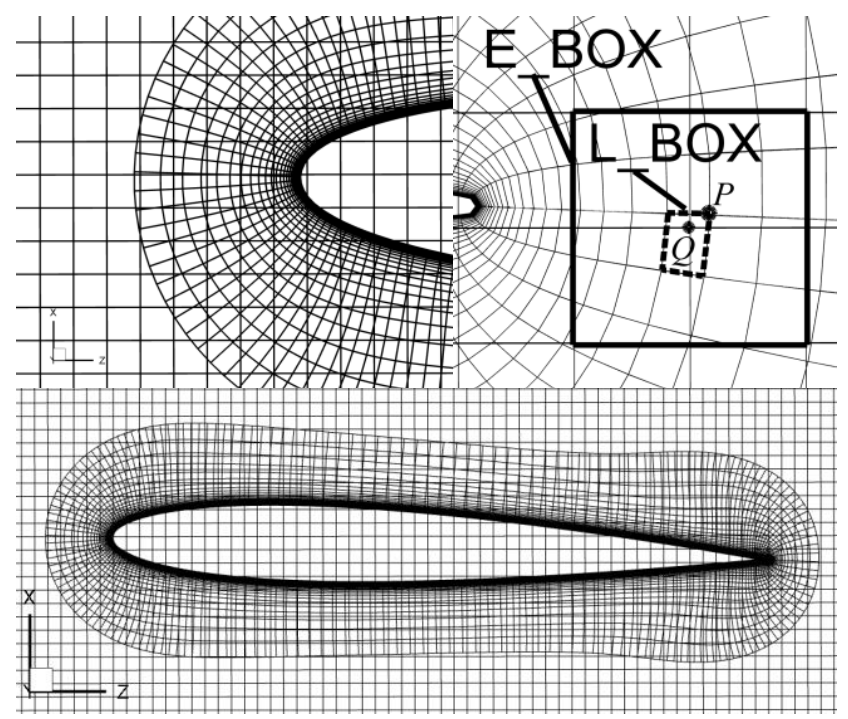

\section{Figure 4 Example for Half-Domain Body-Fitted Mesh Method}

In order to satisfy the conservation law, conservative vector $U$ in Equation 7 is selected for interpolation. The grids of overlapping regions should be of the same order of magnitude on the spatial scale.

Flow solver codes in Fortran95 have been developed. The flow chart of it has been shown in figure 5. This program can be divided into six parts (gray blocks in figure 5) according to their functions: the left-most gray block represents the LSM grid flow result files, and the right-most gray block represents the ECM flow files; The middle four parts, from top to bottom, respectively represent "main computing program", "grid moving program", "grid information interpolation program" and "flow field solver".

Program for the topology enclosure reconstruction of blade tip geometry has also been developed in Fortran95. This program can automatically and quickly generate high-quality half-domain body-fitted blade grids with almost no manual intervention.

The orthogonal solver is used on the Eulerian Cartesian Meshes where the number of mesh points are more. The nonorthogonal solver is used in the body-fitted grids with fewer calculated points. Two sets of solvers will help to improve the 
calculation efficiency. Compared with cartesian meshes, nonorthogonal meshes have to calculate the metric coefficients, and their solver requires significantly more computation. Therefore, two sets of grids are more efficient than one set of non-orthogonal grids.

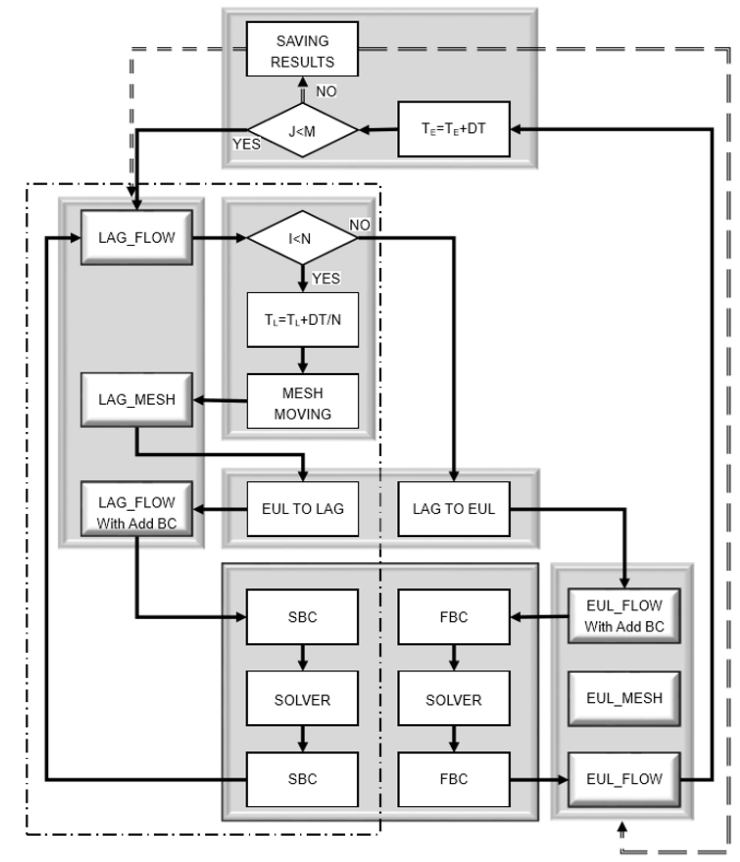

Figure 5 Flow Chart of "HBM-IBM" Fortran Codes

\section{NUMERICAL METHODS}

Governing Equations

Governing equations on the fixed orthogonal Euler meshes are the nondimensionalized Reynolds Averaged Navier-Stokes equations under the Cartesian coordinate $\operatorname{system}(x, y, z, t)$ :

$$
\frac{\partial \boldsymbol{U}}{\partial t}+\frac{\partial \boldsymbol{F}}{\partial x}+\frac{\partial \boldsymbol{G}}{\partial y}+\frac{\partial \boldsymbol{H}}{\partial z}=\frac{\partial \boldsymbol{F}_{v}}{\partial x}+\frac{\partial \boldsymbol{G}_{v}}{\partial y}+\frac{\partial \boldsymbol{H}_{v}}{\partial z}
$$

The vector $\boldsymbol{U}$ is the conserved flux, the vector $\boldsymbol{F}, \boldsymbol{G}, \boldsymbol{H}$ are the inviscid flux terms, and vector $\boldsymbol{F}_{v}, \boldsymbol{G}_{v}, \boldsymbol{H}_{v}$ are the viscous flux terms.

$$
\boldsymbol{U}=\left[\begin{array}{c}
\rho \\
\rho u \\
\rho v \\
\rho w \\
\rho e_{t}
\end{array}\right], \boldsymbol{F}=\left[\begin{array}{c}
\rho u \\
\rho u u+p \\
\rho v u \\
\rho w u \\
\left(\rho e_{t}+p\right) u
\end{array}\right], \boldsymbol{G}=\left[\begin{array}{c}
\rho u \\
\rho u u+p \\
\rho v u \\
\rho w u \\
\left(\rho e_{t}+p\right) u
\end{array}\right], \boldsymbol{H}=\left[\begin{array}{c}
\rho w \\
\rho u w \\
\rho v w \\
\rho w w+p \\
\left(\rho e_{t}+p\right) w
\end{array}\right]
$$

The Reynolds Averaged Navier-Stokes equations on the body-fitted meshes are under the orthogonal coordinate $\operatorname{system}(\xi, \eta, \zeta, \tau)$ :

$$
\frac{\partial \boldsymbol{U}}{\partial \tau}=R_{c}(\boldsymbol{U})+R_{d}(\boldsymbol{U})
$$

Where, $\frac{\partial U}{\partial \tau}$ is the time partial derivative of the conservative vector, $R_{c}(\boldsymbol{U})$ and $R_{d}(\boldsymbol{U})$ are the convection terms and dissipative terms.

$$
\begin{gathered}
R_{c}(\boldsymbol{U})=-J\left[D_{\xi}(\widetilde{\boldsymbol{F}})+D_{\eta}(\widetilde{\boldsymbol{G}})+D_{\zeta}(\widetilde{\boldsymbol{H}})\right] \\
R_{d}(\boldsymbol{U})=J\left[D_{\xi}\left(\widetilde{\boldsymbol{F}}_{v}\right)+D_{\eta}\left(\widetilde{\boldsymbol{G}}_{v}\right)+D_{\zeta}\left(\widetilde{\boldsymbol{H}}_{v}\right)\right] \\
\widetilde{\boldsymbol{F}}=\left(J^{-1} \xi_{x} \boldsymbol{F}+J^{-1} \xi_{y} \boldsymbol{G}+J^{-1} \xi_{z} \boldsymbol{H}\right)+J^{-1} \xi_{t} \boldsymbol{U}
\end{gathered}
$$

In this paper, finite difference method is used for spatial discretization. $\bar{D}$ is used to represent the central-difference operator. $D^{+}$and $D^{-}$respectively denote the forwarddifference operator and backward-difference operator. The subscript $\xi, \eta, \zeta$ denote difference directions. The spatial difference of convective and dissipative terms can be abbreviated as:

$$
\begin{gathered}
D_{\xi}(\widetilde{\boldsymbol{F}})=D_{\xi}^{+}\left(\widetilde{\boldsymbol{F}}^{+}\right)+D_{\xi}^{-}\left(\widetilde{\boldsymbol{F}}^{-}\right) \\
D_{\xi}\left(\widetilde{\boldsymbol{F}}_{v}\right)=\bar{D}_{\xi}\left(\widetilde{\boldsymbol{F}}_{v}\right)
\end{gathered}
$$

\section{Spatial Discretization.}

Because of the strong hyperbolic property of convection terms $R_{c}(\boldsymbol{U})$ in N-S equations, upwind schemes, such as, WENO scheme, which has the characteristics of high discontinuity resolution, high difference accuracy, slight numerical oscillation and small numerical dissipation, should be selected. Therefore, this paper adopts Steger-Warming Flux-vector splitting schemes combined with 5th-order WENO-Z schemes to carry out the spatial dispersion of convection terms.

The 8th-order central difference scheme is used for spatial discretization of dissipative term $R_{d}(\boldsymbol{U})$ due to its dissipative property.

\section{Temporal Discretization}

Explicit three-steps and third-order R-K method is adopted for both steady and unsteady flow simulation:

$$
\begin{aligned}
\boldsymbol{U}^{(0)} & =\boldsymbol{U}^{(n)} \\
\boldsymbol{U}^{(1)} & =\boldsymbol{U}^{(0)}+\Delta \tau \boldsymbol{R}\left(\boldsymbol{U}^{(0)}\right) \\
\boldsymbol{U}^{(2)} & =\frac{3}{4} \boldsymbol{U}^{(0)}+\frac{1}{4} \boldsymbol{U}^{(1)}+\frac{1}{4} \Delta \tau \boldsymbol{R}\left(\boldsymbol{U}^{(1)}\right) \\
\boldsymbol{U}^{(3)} & =\frac{1}{3} \boldsymbol{U}^{(0)}+\frac{2}{3} \boldsymbol{U}^{(2)}+\frac{2}{3} \Delta \tau \boldsymbol{R}\left(\boldsymbol{U}^{(2)}\right) \\
\boldsymbol{U}^{(n+1)} & =\boldsymbol{U}^{(3)}
\end{aligned}
$$

\section{Time Step Determination}

Every explicit time-stepping scheme remains stable only up to a certain value of the time step $\Delta \tau$. To be stable, the time step $\Delta \tau$ has to fulfill the so-called CFL condition. The empirical formula (proposed by Blazek) is:

$$
\Delta \tau \leq \sigma\left(\Delta \tau_{\text {inv }}^{-1}+2 \Delta \tau_{\text {vis }}^{-1}\right)^{-1}, \quad \sigma=0.7 \sim 0.9
$$

Where,

$$
\begin{gathered}
\Delta \tau_{i n v}^{-1}=\left(\frac{\bar{\lambda}_{\xi}}{\Delta \xi}+\frac{\bar{\lambda}_{\eta}}{\Delta \eta}+\frac{\bar{\lambda}_{\zeta}}{\Delta \zeta}\right), \\
\bar{\lambda}_{\xi}=\left|\xi_{x} u+\xi_{y} v+\xi_{z} w\right|+c \sqrt{\xi_{x}^{2}+\xi_{y}^{2}+\xi_{z}^{2}} \\
\Delta \tau_{v i s}^{-1}=\max \left(\frac{4}{3}, \gamma\right)\left(\frac{\mu_{L}}{P_{r_{L}}}+\frac{\mu_{T}}{P_{r_{T}}}\right) \frac{1}{\rho} . \\
\left(\frac{\xi_{x}^{2}+\xi_{y}^{2}+\xi_{z}^{2}}{\Delta \xi^{2}}+\frac{\eta_{x}^{2}+\eta_{y}^{2}+\eta_{z}^{2}}{\Delta \eta^{2}}+\frac{\zeta_{x}^{2}+\zeta_{y}^{2}+\zeta_{z}^{2}}{\Delta \zeta^{2}}\right)
\end{gathered}
$$

\section{Double Global Time Steps}

Since two sets of non-uniform meshes, background meshes and surface meshes, are respectively used to solve the mainstream and boundary layer flows, the two sets of meshes can adopt different global time steps. Small time step $\Delta \tau_{L}$ is 
used on the surface grids, and large time step $\Delta \tau_{E}$ is used on the background Cartesian grids. Every step of the flow field solution is pushed forward in time on the background Cartesian grids, and several steps on the surface grids, so as to improve the computational efficiency. The ratio of $\Delta \tau_{L}$ to $\Delta \tau_{E}$ is suggested as follows:

$$
\Delta \tau_{L} / \Delta \tau_{E}=\sqrt{\Delta \tau_{E}}
$$

\section{Boundary Conditions}

"Non-slip wall boundary condition" is adopted on the solid surface

$\vec{V}_{\text {bou }}=\vec{V}_{\text {wall }}, T_{\text {bou }}=\frac{1}{3}\left(4 T_{2}-T_{3}\right), \rho_{\text {bou }}=\frac{4 \rho_{2} T_{2}-\rho_{3} T_{3}}{3 T_{\text {bou }}}$

Whitfield and Janus's "non-reflection boundary condition" derived from Euler equations based on the characteristic theory is adopted as far-field boundary condition in order to avoid the numerical oscillation.

Subsonic flow inlet:

$$
\left(\begin{array}{c}
p_{b} \\
\rho_{b} \\
u_{b} \\
v_{b} \\
w_{b}
\end{array}\right)=\left(\begin{array}{c}
{\left[p_{\infty}+p_{e}-\rho_{e} c_{e}\left(\vec{V}_{\infty}-\vec{V}_{e}\right) \cdot \vec{n}\right] / 2} \\
\rho_{\infty}+\left(p_{\infty}-p_{b}\right) / c_{e}^{2} \\
u_{\infty}-\left(p_{\infty}-p_{b}\right) n_{x} /\left(\rho_{e} c_{e}\right) \\
v_{\infty}-\left(p_{\infty}-p_{b}\right) n_{y} /\left(\rho_{e} c_{e}\right) \\
w_{\infty}-\left(p_{\infty}-p_{b}\right) n_{z} /\left(\rho_{e} c_{e}\right)
\end{array}\right)
$$

Subsonic flow outlet:

$$
\left(\begin{array}{c}
p_{b} \\
\rho_{b} \\
u_{b} \\
v_{b} \\
w_{b}
\end{array}\right)=\left(\begin{array}{c}
p_{\infty} \\
\rho_{e}+\left(p_{b}-p_{e}\right) / c_{e}^{2} \\
u_{e}+\left(p_{e}-p_{b}\right) n_{x} /\left(\rho_{e} c_{e}\right) \\
v_{e}+\left(p_{e}-p_{b}\right) n_{y} /\left(\rho_{e} c_{e}\right) \\
w_{e}+\left(p_{e}-p_{b}\right) n_{z} /\left(\rho_{e} c_{e}\right)
\end{array}\right)
$$

Turbulence Model.

Turbulence model adopted is the compressible flow S-A model. The Spalart-Allmaras model is "local", robust, converges fast to steady-state, and requires only moderate grid resolution in the near-wall region. WENO difference scheme and $\mathrm{R}-\mathrm{K}$ time scheme were used on the left part of the S-A equation, and central difference scheme was used on the right part.

$$
\begin{aligned}
\frac{D \bar{\rho} \tilde{v}}{D t} & =\underbrace{c_{b 1} \tilde{S} \bar{\rho} \tilde{v}}_{\text {Production }}-\underbrace{\bar{\rho} c_{w 1} f_{w}\left(\frac{\tilde{v}}{d}\right)^{2}}_{\text {Destruction }} \\
& +\underbrace{\frac{1}{\sigma}\left[\nabla \cdot\left(\left(\mu_{L}+\bar{\rho} \tilde{v}\right) \nabla \tilde{v}\right)+c_{b 2} \nabla \tilde{v} \nabla \bar{\rho} \tilde{v}\right]}_{\text {Diffusion }}
\end{aligned}
$$

\section{RESULTS AND DISCUSSION}

\section{Steady Flow of ONERA_M6 Wing}

Eulerian Cartesian Meshes have been shown in figure 6, and Lagrangian Half-Domain Body-Fitted Meshes of ONERA_M6 wing (in figure 7) were automatically generated by our program. Pressure coefficients on seven sections in spanwise direction have been compare with the experimental results by Schmitt, V. and F. Charpin. The results in figure 8 show that this method has high spatial accuracy. Parameters for steady flow of ONERA_M6 wing are prescribed in table 1.
Table 1 Parameters Prescribed for ONERA_M6 Wing

\begin{tabular}{cc}
$M a=0.84, \quad R e=1.172 \times 10^{7}$ \\
Far-field Condition: & $T_{\infty}=306 K, \quad p_{\infty}=101325 \mathrm{~Pa}$ \\
\hline Solid Boundary & $\alpha=3.06^{\circ}, u=0, v=0, w=0$ \\
Condition: & $\partial T / \partial n=0, \partial p / \partial n=0, \tilde{v}=0$ \\
\hline Initial fluid field: & $p=1, \quad T=1, \quad \tilde{v}=0.1 v$ \\
& $u=0, \quad v=0, \quad w=1$ \\
\hline Number of Grid & Euler Meshes: $73 \times 73 \times 154$ \\
Points: & Lagrange Meshes: $145 \times 49 \times 24$
\end{tabular}

Unsteady Flow of NACA0012 airfoil

2-D grids can be seen in figure 4 . Pressure and density distribution are shown in figure 9 . The cyclic change process of lift coefficient on an oscillating airfoil is shown in figure 10. The comparations of pressure distribution and lift coefficients on NACA0012 airfoil with Landon's experiment show that this method has high precision in time and space. Parameters for unsteady flow of NACA0012 airfoil are prescribed in table 2 .

Table 2 Parameters Prescribed for NACA0012 Airfoil

\begin{tabular}{cc} 
Far-field Condition: & $\begin{array}{c}M a=0.755, \quad R e=5.5 \times 10^{6} \\
T_{\infty}=280 K, \quad p_{\infty}=382198 P a\end{array}$ \\
\hline $\begin{array}{c}\text { Solid Boundary } \\
\text { Condition: }\end{array}$ & $k=0.016^{\circ}+2.51^{\circ} \sin (2 \mathrm{kt})$ \\
& $\partial T / \partial n=0, \partial p / \partial n=0, \tilde{v}=0$ \\
\hline Initial fluid field: & $p=1, \quad T=1, \quad \tilde{v}=0.1 v$ \\
\hline Number of Grids & $u=0, \quad v=0, \quad w=1$ \\
Points: & Euler Meshes: $93 \times 164$ \\
& Lagrange Meshes: $201 \times 32$
\end{tabular}

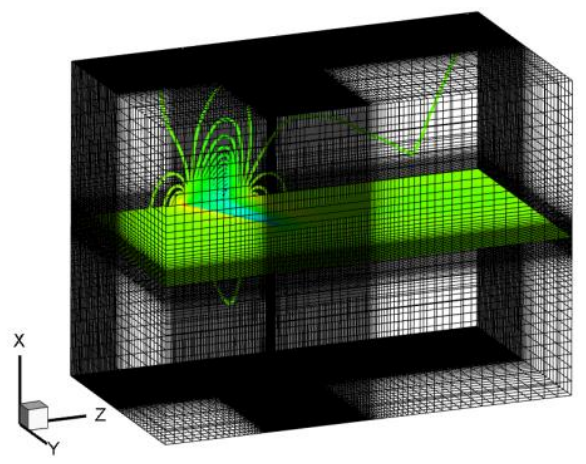

Figure 6 Eulerian Cartesian Meshes (ECM) of ONERA M6 Wing

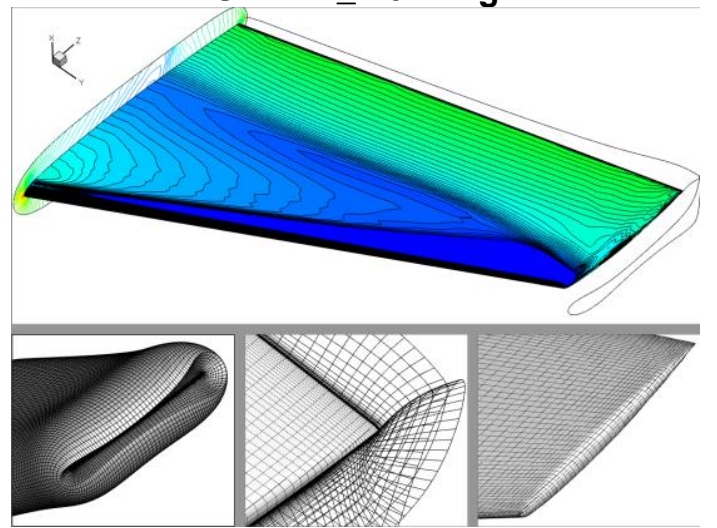

Figure 7 Half-Domain Body-Fitted Meshes and Pressure Distribution of ONER_M6 Wing 


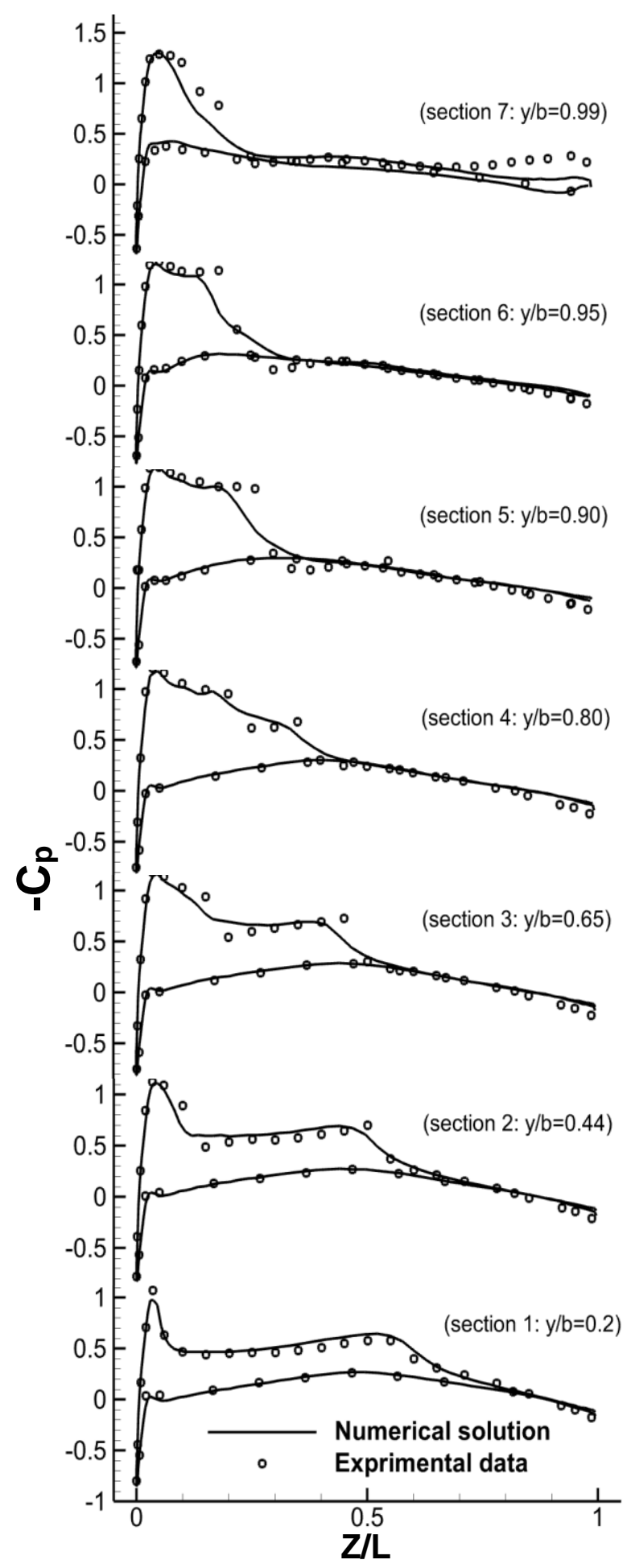

Figure 8 Pressure Distributions at 7 Sections in Spanwise Direction of ONERA_M6 Wing
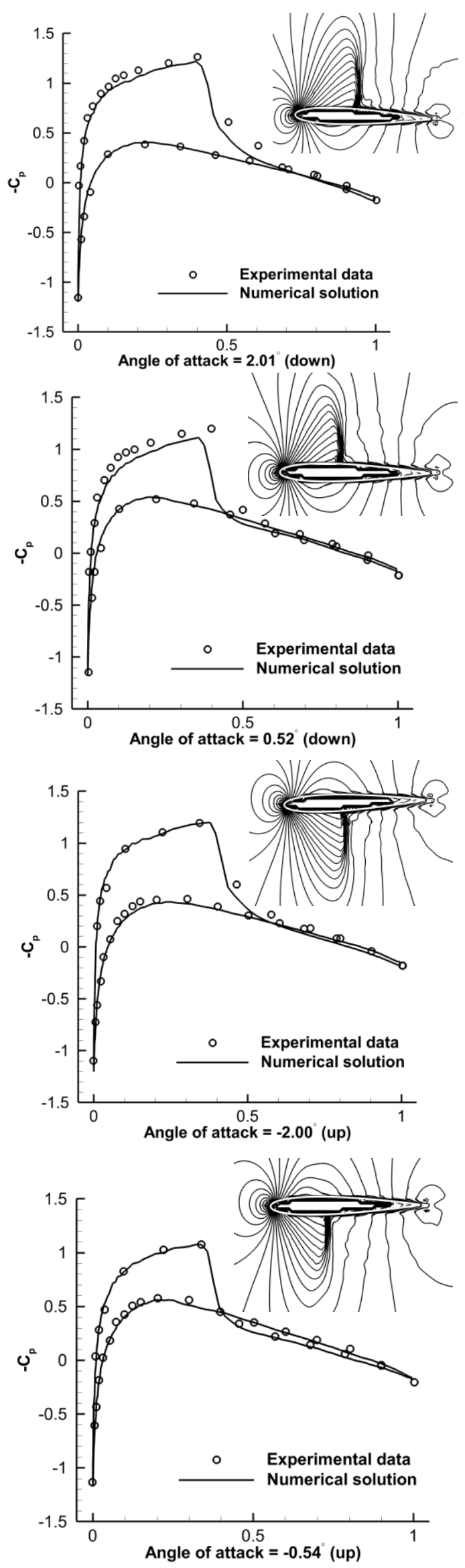

Figure 9 Pressure and Density Distributions at different Angle of Attack of NACA0012 


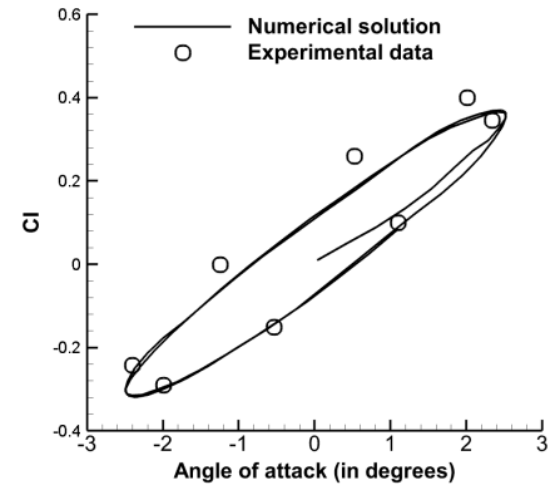

Figure 10 Lift Coefficients versus Angle of Attack in Airfoil Pitching Problem of NACA0012

\section{Unsteady Flow of Propfan}

A single rotor propfan designed by Zhou Yicheng, in the Fluid and Acoustic Engineering Laboratory of BUAA, was numerically simulated. The results were compared with those of NUMECA software. The designed cruising altitude and inlet Mach number of the propfan are $11 \mathrm{~km}$ and 0.8 , and a designed rotation speed is 7246.4 RPM.

The thrust obtained by our program is $255 \mathrm{~N}$ (while $280 \mathrm{~N}$ by NUMECA software). It fully demonstrates that the program has the ability to simulate the complex viscous flow of a high-speed rotating turbomachinery. Due to the limited computing capacity of hardware and relatively coarse grids, there is still much room for improvement in the accuracy of the results. Parameters for unsteady flow of propfan are prescribed in table 3 .

Half-domain Body-fitted Lagrangian meshes for propfan blades are automatically generated by HBM-IBM Program Codes (Shown in figure 11, 12). Swirl distribution in Eulerian meshes are shown in figure 13, which shows that Eulerian meshes can accurately capture and simulate the flow near the moving blades. Static pressure distribution on the surface of propfans can be seen in figure 14 .

Table 3 Parameters prescribed for Propfan

\begin{tabular}{ccc} 
Far-field Condition: & $M a=0.8, \quad R e=1.88 \times 10^{6}$ \\
& $T_{\infty}=216.7 \mathrm{~K}, \quad p_{\infty}=22700 \mathrm{~Pa}$ \\
\hline Solid Boundary & $n=7246.4 \mathrm{r} / \mathrm{min}$ \\
Condition: & $\partial T / \partial n=0, \partial p / \partial n=0, \tilde{v}=0$ \\
\hline \multirow{2}{*}{ Initial fluid field: } & $p=1, \quad T=1, \quad \tilde{v}=0.1 v$ \\
& $u=0, \quad v=0, \quad w=1$ \\
\hline Number of Grids & Euler Meshes: $165 \times 165 \times 104$ \\
Points: & Lagrange Meshes: $143 \times 51 \times 28 \times 8$
\end{tabular}

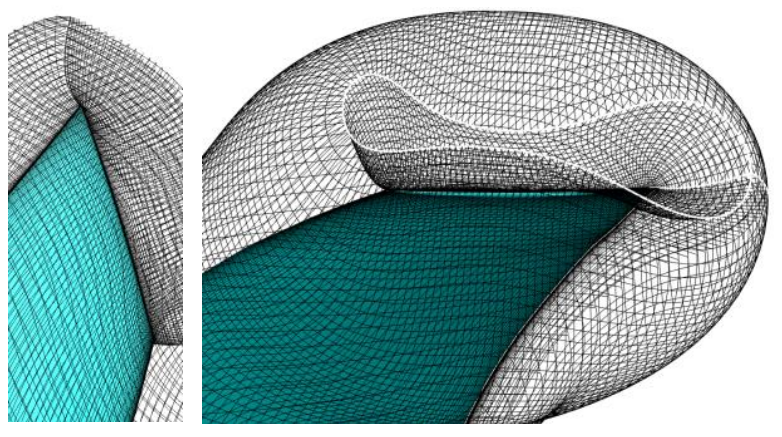

Figure 11 Blade Grids Automatically Generated by HBM-IBM Program Codes

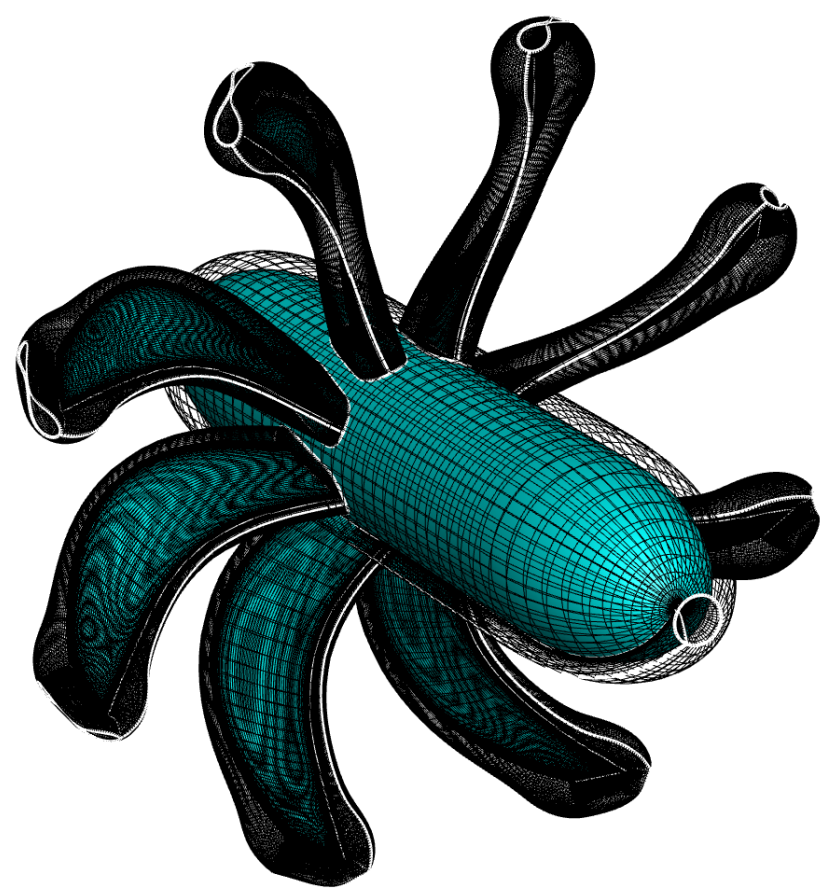

Figure 12 Half-domain Body-fitted Lagrangian Grids Generated by HBM-IBM Program Codes

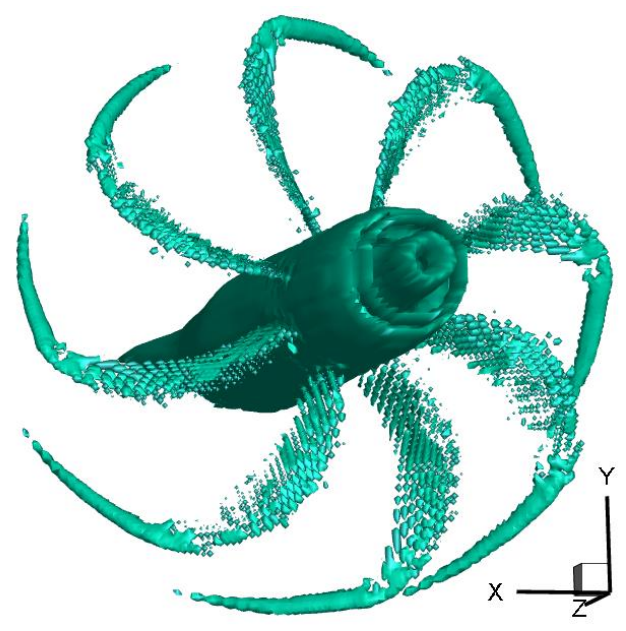

Figure 13 Swirl Distribution in Eulerian Meshes 


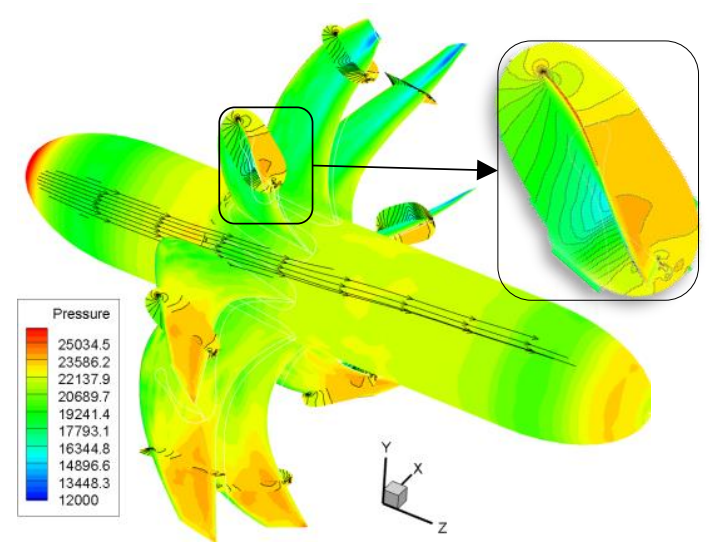

Figure 14 Pressure Distribution on Blades

\section{CONCLUSIONS}

The advantage of the method in this paper is to simplify the complexity of grid generation as much as possible under the premise of high computational accuracy, but not to be more accurate or faster than other numerical methods.

The similarity between this method and the common overlapping grid method is that the necessary information is exchanged between different grids by spatial interpolation of physical flux. The difference is that there are primary and secondary grids in this method. The primary cartesian grids provide information of the whole flow field, and the bodyfitted grids provides accurate flow information near the object surface. In one time advancement step, the flow fields of the two sets of grids are updated alternately, instead of being updated independently like other overlapping grid methods.

This method is essentially a kind of implicit force source term immersed boundary method, which combines the overset grid technology with locally densified near-wall grids. Compared with the classical immersed boundary method, this method has the advantages of auto-generation of computational grids, simple process of finding contribution units, high calculation efficiency and high spatial and temporal simulation accuracy. It is very easy to implement high order difference schemes and turbulence models via Half-Domain Body-Fitted Mesh Method, which can be widely used in numerical simulation for the flows over aero-propeller, propfan and other turbo-machines.

\section{References}

Anderson, D., Tannehill, J., Pletcher, R. (2013). Computational Fluid Mechanics and Heat Transfer. Boca Raton: CRC Press, https://doi.org/10.1201/b12884

Blazek, J. (2007). Spatial Discretisation. In Computational Fluid Dynamics: Principles and Applications. Elsevier.

\section{https://doi.org/10.1016/b978-008043009-6/50006-6}

Borges, R., Carmona, M., Costa, B., \& Don, W. S. (2008).

An improved weighted essentially non-oscillatory scheme for hyperbolic conservation laws. Journal of Computational Physics, 227(6), 3191-3211.

https://doi.org/10.1016/j.jcp.2007.11.038
Chiu, I.-T., \& Meakin, R. (2013). On automating domain connectivity for overset grids. American Institute of Aeronautics and Astronautics (AIAA).

https://doi.org/10.2514/6.1995-854

Courant, R., Friedrichs, K., \& Lewy, H. (1967). On the Partial Difference Equations of Mathematical Physics (Republished). IBM Journal of Research and Development, 11(2), 215-234. https://doi.org/10.1147/rd.112.0215

Deck, S., Duveau, P., D’Espiney, P., \& Guillen, P. (2002). Development and application of Spalart-Allmaras one equation turbulence model to three-dimensional supersonic complex configurations. Aerospace Science and Technology, 6(3), 171-183.

https://doi.org/10.1016/S1270-9638(02)01148-3

Guillard, H., \& Farhat, C. (2000). On the significance of the geometric conservation law for flow computations on moving meshes. Computer Methods in Applied Mechanics and Engineering, 190(11-12), 1467-1482.

https://doi.org/10.1016/S0045-7825(00)00173-0

Hoffmann, K. A., \& Chiang, S. T. (2000). Computational Fluid Dynamics Volume I. International Journal of Computational Fluid Dynamics.

https://doi.org/10.1111/j.1525-1594.2010.01087.x

Landon, R. H. (1982). NACA0012 oscillation and transient pitching," in Compendium of Unsteady Aerodynamic Measurements, Advisory Report 702.

Mittal, R., Dong, H., Bozkurttas, M., Najjar, F. M., Vargas, A., \& von Loebbecke, A. (2008). A versatile sharp interface immersed boundary method for incompressible flows with complex boundaries. Journal of Computational Physics, 227(10), 4825-4852.

https://doi.org/10.1016/j.jcp.2008.01.028

Peskin, C. S. (2010). The immersed boundary method. In Acta Numerica 2002 (pp. 479-518). Cambridge University Press.

https://doi.org/10.1017/cbo9780511550140.007

SPALART, P., \& ALLMARAS, S. (2013). A oneequation turbulence model for aerodynamic flows. American Institute of Aeronautics and Astronautics (AIAA).

https://doi.org/10.2514/6.1992-439

Steger, J. L., \& Warming, R. F. (1981). Flux vector splitting of the inviscid gasdynamic equations with application to finite-difference methods. Journal of Computational Physics, 40(2), 263-293.

https://doi.org/10.1016/0021-9991(81)90210-2

Schmitt, V. and F. Charpin,(1979). Pressure Distributions on the ONERA-M6-Wing at Transonic Mach Numbers, Experimental Data Base for Computer Program Assessment. Report of the Fluid Dynamics Panel Working Group 04, AGARD AR 138, May.

Tseng, Y. H., \& Ferziger, J. H. (2003). A ghost-cell immersed boundary method for flow in complex geometry. Journal of Computational Physics, 192(2), 593-623. https://doi.org/10.1016/j.jpp.2003.07.024

WHITFIELD, D. (2013). Three-dimensional unsteady Euler equations solution using flux vector splitting. American Institute of Aeronautics and Astronautics (AIAA). https://doi.org/10.2514/6.1984-1552 